\title{
Les aides financières aux étudiants
}

Tendances et réalités dans l'Union européenne

Financial help for students. Trends and realities in the European Union

Las ayudas financieras a los estudiantes. Usos y realidades en la Unión Europea

Arlette Delhaxhe, Anne Godenir et Laurent Deutsch

\section{OpenEdition \\ Journals}

Édition électronique

URL : http://journals.openedition.org/ries/2987

DOI : 10.4000/ries.2987

ISSN : 2261-4265

Éditeur

Centre international d'études pédagogiques

Édition imprimée

Date de publication : 1 juin 1999

Pagination : 83-91

ISSN : 1254-4590

Référence électronique

Arlette Delhaxhe, Anne Godenir et Laurent Deutsch, «Les aides financières aux étudiants », Revue

internationale d'éducation de Sèvres [En ligne], 22 | 1999, mis en ligne le 13 mai 2013, consulté le 03 mai 2019. URL : http://journals.openedition.org/ries/2987 ; DOI : 10.4000/ries.2987

Ce document a été généré automatiquement le 3 mai 2019.

(c) Tous droits réservés 


\title{
Les aides financières aux étudiants
}

\author{
Tendances et réalités dans l'Union européenne \\ Financial help for students. Trends and realities in the European Union \\ Las ayudas financieras a los estudiantes. Usos y realidades en la Unión Europea
}

\author{
Arlette Delhaxhe, Anne Godenir et Laurent Deutsch
}

1 Bon nombre d'ouvrages sont consacrés à la question du financement de l'éducation en général et à celui de l'enseignement supérieur en particulier. Au-delà de l'élaboration et de la comparaison des indicateurs statistiques de dépenses, les études disponibles examinent essentiellement ce qu'on appelle couramment le « retour de l'investissement en capital humain ». Elles tentent de mettre en relation la "production» du système éducatif avec son coût. Cette dernière mesure ne peut se faire sans prendre en compte les objectifs poursuivis par le système de financement mis en place. Se pose, en effet, à celui qui veut comprendre et bien interpréter le financement de l'éducation, la difficile question de la part des interventions financières publique et privée, envisagée simultanément du double point de vue de l'efficacité et de l'équité. Il importe d'élargir la logique strictement économique et statistique afin d'analyser le fonctionnement des systèmes (critères d'octroi, organes responsables des décisions de dépenses, nombre d'élèves et d'étudiants concernés, etc.) et les situer dans le cadre des différentes cultures et de leur histoire. C'est dans cette perspective qu'Eurydice $^{1}$ a mené une étude approfondie des aides financières publiques aux étudiants de l'enseignement supérieur.

Le présent article synthétise les éléments essentiels qui ressortent de l'ouvrage publié dans la nouvelle série Questions clés de l'éducation en Europe ${ }^{2}$. L'étude a été réalisée par l'unité européenne d'Eurydice en étroite collaboration avec les unités nationales de son réseau pour la partie descriptive et un réseau d'experts nationaux dans le domaine de l'économie de l'éducation pour la partie contextuelle. Toute l'analyse comparative a été rédigée sur la base des rapports détaillés de ces partenaires nationaux. L'ouvrage contient une analyse approfondie des différentes formes d'aide financière publique a permettant aux jeunes de mener des études. Les composantes, d'abord examinées isolément, sont intégrées en quelques modèles qui se dégagent de l'analyse et caractérisent l'ensemble des pays de l'Union européenne et de l'Espace économique européen. L'originalité de 
cette étude réside sans nul doute dans sa dimension historique et contextuelle. La compréhension des fondements idéologiques et des objectifs poursuivis par la mise en place des systèmes d'aide financière ainsi que l'analyse des facteurs qui en ont influencé l'évolution sont au cœur de l'analyse. Les indicateurs statistiques développés sur la base des données fiables disponibles sont systématiquement analysés à la lumière des situations des contextes nationaux.

3 La problématique de l'aide financière aux étudiants peut paraitre à première vue réduite mais, dès qu'on analyse ses différents aspects, on s'aperçoit rapidement que les composantes à prendre en considération sont multiples. On distinguera les aides sous forme de bourses et de prêts, les aides aux parents d'étudiants que constituent les avantages fiscaux et les allocations familiales, les services subventionnés et autres avantages sociaux. Du point de vue quantitatif, la problématique mérite également qu'on $s^{\prime} y$ intéresse. Le nombre d'étudiants ${ }^{3}$ concernés par les bourses et prêts est assez élevé (on comptait $30 \%$ environ en moyenne d'étudiants boursiers dans l'Union européenne en 1996-1997 et $12 \%$ de bénéficiaires d'un prêt). Les montants dépensés chaque année par les pouvoirs publics en la matière constituent, en moyenne pour l'Union, près d'un cinquième $(17 \%)$ de la part des dépenses publiques dans l'enseignement supérieur : ce qui correspond à un budget global moyen de près de douze milliards d'euros ${ }^{4}$.

\section{Modèles d'aides et principes de société}

4 L'analyse comparative met en évidence une variation importante entre les pays tant dans les composantes du système d'aide (formes d'aide, conditions d'octroi et de maintien) que dans les budgets alloués et les pourcentages d'étudiants aidés. Dans certains pays, les aides sont ciblées sur un nombre limité d'étudiants retenus en fonction du revenu des parents et dans d'autres pays, elles sont généralisées et quasi universelles. Elles peuvent aussi être réservées aux étudiants principalement sous la forme de bourses et/ou de prêts ou être élargies aux familles sous la forme d'avantages fiscaux et/ou d'allocations.

5 Ces variations sont étroitement liées à la position des pays sur deux grands axes en lien avec des principes de société :

6 - le degré de dépendance des étudiants vis-à-vis de leurs parents qui se traduit soit par l'indépendance financière des jeunes à l'égard de leurs parents, soit par la responsabilité financière des parents à l'égard de leur enfant ;

7 - l'importance du devoir d'intervention de l'État qui se traduit soit par la contribution privée dans le coût de l'enseignement supérieur, soit par sa prise en charge intégrale par les pouvoirs publics.

8 La position des pays par rapport à ces axes détermine très fortement les types d'aide mis en place mais aussi le public visé par ces aides. Il importe de souligner que la position d'un pays sur un axe est indépendante de sa position sur l'autre.

9 Les pays nordiques se caractérisent par l'indépendance financière des jeunes par rapport à leurs parents et par la gratuité d'accès aux études. Aucune aide n'est accordée aux familles pour couvrir la charge d'un enfant étudiant. Seuls les étudiants peuvent bénéficier de bourses et/ou de prêts. Cette forme unique de soutien financier est accordée dans tous ces pays à une grande partie de la population étudiante (plus de la moitié d'entre eux au moins) et le montant est supposé couvrir globalement le coût de la vie. 
10 L'Allemagne, la Grèce et l'Autriche ont en commun avec les pays nordiques le principe de gratuité d'accès à l'enseignement supérieur mais ils s'en différencient par le principe de responsabilité des familles à l'égard de leurs enfants. En conséquence, les familles disposent pour leurs jeunes au-delà de dix-huit ans et toujours étudiants des mêmes aides que celles accordées aux parents de jeunes enfants. Des aides sont en outre accordées aux étudiants sous forme de bourses et/ou de prêts en fonction des revenus de leurs parents. Ces aides sont donc ciblées sur les plus démunis et le nombre de leurs bénéficiaires est moins élevé que dans le premier groupe. Les aides accordées aux familles ont davantage un caractère universel.

11 La Belgique, la France et les pays latins se distinguent des deux groupes précédents par l'existence de frais de scolarité et/ou d'inscription payés par l'étudiant à l'établissement d'enseignement supérieur et, donc, par le principe de la contribution privée au coût de l'enseignement mais ces pays adhèrent tous au principe de responsabilité des parents et offrent des aides familiales sous forme d'avantages fiscaux et/ou d'allocations. Comme dans les pays germaniques, les aides allouées aux familles y sont universelles et les aides accordées aux étudiants sont fonction du niveau de revenus des parents. Dans ces pays, pour les étudiants de milieux défavorisés, des aides au paiement des frais d'inscription s'ajoutent à l'octroi des bourses et sont accordées généralement dans les mêmes conditions.

Deux pays (Pays-Bas et Royaume-Uni) se singularisent par leur position intermédiaire quant au principe de la responsabilité des familles. En effet, les parents ne reçoivent pas d'aides pour leur enfant étudiant, ce qui reflète la volonté de l'émancipation des jeunes et l'adhésion au principe de l'indépendance financière. Cependant, une partie des aides financières aux étudiants reste accordée en fonction des revenus des parents. Dans ces deux pays, des droits de scolarité sont payés aux établissements par les étudiants avec des mécanismes d'aides.

13 Au-delà de ces grandes distinctions entre pays, basées uniquement sur la différenciation des composantes, une attention particulière doit être accordée aux modalités de mise en œuvre de certaines aides. En effet, pour une même forme d'aide, des différences s'observent entre les pays tant dans les conditions d'octroi et de maintien que dans les sommes allouées. Ces variations peuvent avoir des conséquences importantes sur l'attrait de l'aide pour l'étudiant et sur le support financier réel qu'elle apporte à celui qui manque de ressources. Deux types d'aides méritent particulièrement d'être examinés dans ce contexte: les prêts et les aides aux familles accordées sous forme d'avantages fiscaux.

14 En matière de prêts, les conditions de remboursement (taux d'intérêts, durée du remboursement, montant des traites en relation avec les revenus, etc.), peuvent engendrer un endettement parfois considérable des étudiants diplômés, néfaste tant pour le diplômé que pour l'État. En cas de défaut de remboursement, les économies visées par les pouvoirs publics par l'augmentation de la contribution privée attendue de la mise en place d'un système de prêts ou des modifications de ses paramètres financiers ne sont pas réalisées.

15 En matière d'aides aux familles, dans certains pays, les avantages fiscaux sont accordés de manière égale à toutes les familles d'étudiants sous la forme de crédits d'impôts, c'est-àdire d'un montant forfaitaire sans lien avec le niveau de revenus. Dans d'autres systèmes, les déductions fiscales sont calculées sur la base des dépenses réelles faites dans le cadre 
du coût des études et/ou déterminent une économie d'impôt proportionnelle aux revenus. Dans ce cas, plus la famille dépense pour les études ou plus elle a un revenu élevé, plus elle réalise une économie d'impôt importante. Ce phénomène ne manque pas d'interpeller ceux pour qui l'objectif principal d'un système d'aide financière est de permettre l'accès des plus démunis à l'enseignement supérieur.

\section{Tendances et contraintes}

$\mathrm{Au}$ cours de ces trente dernières années, peu de changements "structurels " sont intervenus dans les systèmes d'aide financière : les réformes et adaptations nécessaires aux situations nouvelles ont été réalisées dans les limites des composantes existantes. Les changements profonds avec modification de composantes sont rares. On les observe principalement au Royaume-Uni (très récemment) et aux Pays-Bas.

Pour transformer radicalement un système d'aide financière, une vision autre de la société (émancipation des jeunes, principe d'égalité d'accès et devoir d'intervention de l'État) ainsi que des objectifs nouveaux doivent être à la clé du changement.

Parmi les principes de base présents au moment de la mise en place des systèmes d'aides contemporains qui se sont révélés particulièrement stables, on citera le principe de la responsabilité des familles (parfois inscrit dans la Constitution) et celui de la gratuité de l'enseignement.

19 Seuls les pays nordiques ont évolué vers l'indépendance financière complète des jeunes à l'égard de la famille. Ce principe était déjà pleinement présent en Islande depuis longtemps. Dans les autres pays nordiques, les aides étaient au départ accordées en fonction des revenus familiaux. Elles le sont aujourd'hui en fonction des revenus de l'étudiant. L'évolution de ces pays dans ce sens s'explique en partie par l'absence d'aides aux familles pour les jeunes de plus de dix-huit ans. Après de nombreuses années de préparation et de débats, les Pays-Bas ont révolutionné leur système par l'abolition des aides aux familles et la mise en place d'une bourse de base universelle. On peut donc affirmer qu'ils ont évolué vers le principe de l'indépendance financière des jeunes. Cependant, en introduisant dans le système une bourse supplémentaire octroyée en fonction du revenu de leurs parents, la dépendance des jeunes à l'égard de leur famille a été partiellement conservée. Ailleurs, le principe de la responsabilité des familles est resté fort, même si des débats ont été menés dans certains pays sur cette question.

En ce qui concerne la gratuité de l'enseignement, elle a été maintenue partout où les droits de scolarité étaient absents au moment de la mise en place du système d'aide contemporain. Seuls deux Länder en Allemagne ont très récemment introduit une réglementation visant l'augmentation de droits administratifs jusqu'ici insignifiants. La contribution privée au coût des études est aussi récente au Royaume-Uni. Avant 1998, les frais de scolarité n'étaient pas payés par les étudiants mais étaient pris en charge directement par les autorités publiques. Aujourd'hui, ils sont partiellement payés par les étudiants en fonction du revenu des parents. Partout ailleurs où les frais d'inscription et de scolarité existaient dans les années soixante-dix, ils ont été maintenus et ont, en général, fortement augmenté. Ces augmentations ont systématiquement été accompagnées d'un système d'aides pour les étudiants démunis sous forme de réduction, d'exonération ou de complément à la bourse. L'augmentation des frais d'inscription ou de scolarité a généralement provoqué des débats et contestations atténuées dans certains 
cas par la mise en place de déduction fiscale pour les familles qui paient des frais à l'inscription. Dans ce contexte, l'évolution de l'Irlande est particulière. La forte augmentation des frais de scolarité dans les années quatre-vingt s'était accompagnée de l'introduction d'une mesure de déduction fiscale pour les parents qui accordaient une rente à leur enfant. Cette mesure fiscale avait engendré des dépenses importantes étant donné le nombre élevé de parents qui utilisaient cet avantage fiscal. Elle a été abolie récemment pour des raisons d'équité simultanément avec la suppression des droits de scolarité pour la majorité des étudiants.

$21 \mathrm{Au}$ cours des trois dernières décennies, les systèmes d'aide financière dans l'enseignement supérieur des pays de l'Union n'ont pas tous été affectés par des facteurs démographiques, politiques et socio-économiques identiques. De plus, lorsque les mêmes facteurs sont intervenus dans plusieurs pays, ils n'ont pas toujours modifié les systèmes d'aides ou les ont influencés différemment. En fait, les décisions prises par les décideurs politiques ont été étroitement déterminées par les principes de base sur lesquels reposent les systèmes d'aides au départ, par les objectifs poursuivis et les composantes existantes.

Ainsi, la mise en place de prêts s'avère une entreprise difficile là où les bourses constituent traditionnellement la forme d'aide unique. La plupart des tentatives de mise en place ont peu de succès voire même ont avorté, soit parce que les prêts sont très peu utilisés par les étudiants, soit parce que les banques se sont montrées réticentes à offrir des prêts. Seul, le Royaume-Uni semble avoir réussi cette conversion dans un contexte de promotion de l'indépendance et de la responsabilité financière des étudiants. D'une manière générale, même dans les pays où les prêts ont toujours existé, les étudiants évitent d'emprunter lorsque cela est possible. Souvent, ils préfèrent recourir au travail rémunéré. Cela engendre une prolongation des études et donc une dépense au niveau de l'enseignement supérieur plus élevée pour les pouvoirs publics. La recherche de l'équilibre entre la part du prêt (y compris le calcul du taux d'intérêt et des conditions de remboursement) et la part de la bourse est au cœur des préoccupations des pays nordiques qui luttent sans cesse contre l'endettement des étudiants après les études et/ ou contre le travail des étudiants pendant les études. Dans ces pays où le prêt a toujours été une composante de l'aide, la réponse quasi générale à ces deux problèmes a été d'augmenter la part de la bourse.

La suppression des aides accordées à tous les parents d'étudiants au profit d'une aide unique octroyée à tous les étudiants indépendamment des revenus des parents - réalisée aux Pays-Bas en 1986 - a fait seulement l'objet de débats dans plusieurs autres pays. Les freins à cette transformation des systèmes sont doubles. Il s'agit du principe de la responsabilité des familles et du coût que représente une telle opération. Aux Pays-Bas, pour des raisons financières, le système évolue actuellement vers une diminution de la part universelle de l'aide et vers un renforcement du soutien financier aux étudiants issus de milieux défavorisés. La caractéristique universelle des aides familiales peut également être remise en question. Ainsi, en Autriche, des coupes budgétaires importantes ont été réalisées dans les aides aux familles en liant leur octroi et maintien à la réussite aux études.

En ce qui concerne l'évolution du devoir de réussite pour l'octroi et le maintien d'une bourse et/ou d'un prêt, on observe une certaine convergence entre les pays. En effet, les pays les plus flexibles sur cette question ont renforcé leurs exigences et, inversement, ceux qui n'accordaient aucun droit à l'échec pour assurer le maintien de la bourse ont pris des mesures autorisant une prolongation de l'aide. La liaison de l'octroi de l'aide à la 
réussite apparait d'autant plus étroite que les aides sont généreuses, c'est-à-dire qu'elles sont accordées à une proportion importante d'étudiants avec une part de bourse élevée. En effet, il importe de responsabiliser les étudiants pour éviter qu'ils ne se servent des deniers publics à d'autres fins que de mener à bien leurs études.

Enfin, au-delà des divergences entre les pays dans les composantes de départ et dans les principes de base sur lesquels reposent les systèmes d'aides, trois grandes tendances se dessinent dans l'évolution du public visé par les aides.

La première se caractérise par le maintien ou la confirmation de la répartition égalitaire des aides avec distribution égale des aides aux étudiants indépendamment des revenus de leurs parents. Elle est observée dans les pays nordiques, ainsi qu'en Grèce et en Autriche. La deuxième tendance correspond à une redistribution des aides vers les plus démunis à partir de la contribution des familles aisées. Sans rompre avec la tradition des aides aux familles, la redistribution peut prendre la forme d'une suppression de toutes les déductions fiscales qui engendrent une progressivité dans l'économie de l'impôt et qui profitent donc davantage aux plus nantis. À cette mesure, peut s'ajouter l'augmentation des aides ciblées sur les étudiants démunis. Cette évolution s'observe surtout en Belgique, en Irlande, en Italie, aux Pays-Bas et au Portugal. Quelques pays ont conservé un profil de double polarisation dans la répartition de leurs aides. Les aides sous forme de bourses sont ciblées vers les étudiants démunis mais les aides aux familles contiennent un élément favorisant les plus nantis. C'est le cas en Allemagne, en France, au Luxembourg et au Liechtenstein.

\section{Au cœur du débat}

Trois questions fondamentales devraient être au cœur de toutes les réformes ou débats en matière d'aide financière aux étudiants de l'enseignement supérieur: quels sont les principes de base sur lesquels le système repose? Quels sont les objectifs poursuivis? Quels sont les facteurs qui imposent un changement? Les composantes des formes d'aides, le public visé par ces aides ainsi que les moyens financiers nécessaires dépendent des réponses apportées à ces questions.

Les problématiques de l'égalité d'accès et de l'équilibre entre intervention de l'État et contribution privée sont toujours au cœur des tensions qui animent les débats en matière d'aide financière aux étudiants. Ceux qui plaident en faveur des frais d'inscription aux études et/ou en faveur de la mise en place de systèmes de prêts remboursables trouvent généralement leurs arguments dans le principe de la contribution privée vu le bénéfice individuel engendré par le diplôme.

29 Ceux qui plaident pour la gratuité d'accès et l'octroi d'aides non remboursables s'inquiètent de la réalisation du principe d'égalité d'accès, voire de l'équilibre social du recrutement dans l'enseignement supérieur.

Quelques pays (Italie, Portugal, Royaume-Uni) viennent d'introduire ou d'augmenter considérablement les droits de scolarité. Cette réforme s'accompagne d'une compensation financière pour les étudiants issus de familles plus démunies (soit par réduction ou exemption du paiement, soit par augmentation de la bourse). L'objectif est clair : augmenter la contribution privée au coût des études en respectant le principe de l'égalité d'accès. Il est trop tôt pour mesurer les effets de ces mesures récentes. Seule l'évolution des prochaines années du budget de l'enseignement et de l'accès aux études 
permettra d'examiner comment les objectifs poursuivis par ces mesures se réalisent effectivement. Les débats sur l'augmentation ou la mise en place de frais d'inscription à charge des étudiants ne peuvent ignorer une variable importante dans les pays où des avantages fiscaux existent: l'économie réalisée d'un côté par les pouvoirs publics par l'augmentation de la contribution privée aux coûts des études se réduit lorsque des possibilités de déductions fiscales sont offertes aux familles en fonction de leurs dépenses réalisées en paiement des droits de scolarité.

$\mathrm{Au}$ terme de cette étude, une question reste posée : l'augmentation de la contribution privée et les contraintes imposées par les pouvoirs publics constitueront-elles des obstacles à la réalisation du principe d'égalité d'accès recherchée partout?

L'expérience nordique nous enseigne que l'augmentation de la contribution privée, via les prêts dans un système égalitaire, accroît l'endettement des diplômés et a des répercussions sur les budgets publics étant donné les défauts de remboursement. Ce phénomène a conduit la plupart des pays qui ont testé cette option à réviser leurs décisions. L'augmentation de la contribution privée via les droits de scolarité et les prêts dans des systèmes redistributifs (où les familles aisées paient pour les familles défavorisées) n'a pas encore eu le temps de faire ses preuves. Seules les réactions de la population concernée dans les prochaines années permettront de répondre.

On ne peut conclure une étude sur les aides financières aux étudiants sans s'interroger sur les mesures mises en œuvre en lien avec le principe du devoir de réussite. Ce principe s'est accentué ces dernières années en raison de la volonté politique d'accroître la responsabilité des étudiants et de réduire la durée des études et partant le coût de l'éducation. Les mesures touchent particulièrement le progrès exigé dans les études. Elles peuvent prendre plusieurs formes mais toutes limitent d'une manière ou d'une autre le droit de l'étudiant de recevoir une aide lorsqu'il prolonge ses études au-delà de la durée dite normale. Sans nier les effets que ces mesures peuvent avoir sur la motivation des étudiants à réussir, on peut craindre qu'elles ne constituent un frein à l'accès des jeunes issus de milieux défavorisés et qu'en conséquence, elles contredisent le principe d'égalité d'accès. En effet, déjà peu enclins à prolonger leurs études pour des raisons économiques et culturelles, ces jeunes se voient ainsi confrontés à une barrière supplémentaire : l'obligation de réussite rapide. Comme cela a été souligné ci-dessus, on observe un mouvement convergent entre les pays qui étaient encore récemment extrêmement divisés sur cette question. À nouveau, l'évolution des prochaines années permettra de mettre en évidence les effets de telles mesures sur l'accès aux études, mais surtout sur l'abandon dont on commence à mesurer aujourd'hui l'importance.

Plusieurs pays ont mis en place un observatoire de la vie étudiante et mènent des enquêtes sur des aspects divers de la situation de cette population. On ne peut que se réjouir de telles initiatives. Elles devraient permettre de dépasser les analyses des décisions politiques et d'enrichir les données statistiques sur les dépenses macroéconomiques actuellement disponibles. Elles offrent, en effet, la possibilité d'examiner sur le terrain à la fois les effets concrets des aides et les représentations sociales des jeunes sur cette question (crainte de l'endettement, bénéfice personnel, critère de réussite, etc.). 


\section{NOTES}

1. Réseau européen d'information sur l'éducation mis sur pied en 1980 à l'initiative de la Commission européenne.

2. Commission européenne/Eurydice, Les aides financières aux étudiants dans l'Union européenne, tendances et débats, volume I, série Questions Clés de l'éducation en Europe, Luxembourg, Office des publications, 1999.

3. L'étude se centre surtout sur les étudiants pour une première qualification. Les aides pour doctorat ou troisième cycle sont peu prises en compte et les aides données en fonction du mérite ne sont pas analysées.

4. Ce montant est sous-estimé puisque il ne prend pas en compte les autres formes d'aides telles que les aides aux familles, les services (repas ou transport) ou encore les autres formes d'avantages sociaux subventionnés par l'État pour les étudiants.

\section{RÉSUMÉS}

Élaboré à partir d'une étude intitulée Les aides financières aux étudiants dans l'Union européenne, tendance et débats, cet article permet d'appréhender les composantes multiples qui sont à prendre en considération dans ce domaine selon les pays de l'Union européenne observés. Outre les principes de base sur lesquels reposent les différents systèmes d'aides et leurs objectifs, une analyse des données qui imposent une évolution est présentée.

This article, based on a study entitled Financial help for students in the European Union, trends and issues, brings out the various components which have to be taken into account in this domain according to the country considered in the European Union. In addition to the basic principles on which the different systems of help and their objectives are founded, an analysis of data is presented which will make changes necessary.

Basado en un estudio titulado Las ayudas financieras a los estudiantes en la Unión Europea, usos y debates, este artículo permite abarcar las múltiples componentes que deben considerarse al estudiar este dominio según los países de la Unión Europea que se analicen. Además de los principios de base en los que descansan los diferentes sistemas de ayudas y sus metas, se presenta un análisis de los datos que imponen una evolución.

\section{INDEX}

Index géographique : Europe

Mots-clés : allocation financière, bourse d'études, enseignement supérieur, étudiant, politique éducative 


\section{AUTEURS}

\section{ARLETTE DELHAXHE}

Unité européenne d'Eurydice, Bruxelles, Belgique ANNE GODENIR

Unité européenne d'Eurydice, Bruxelles, Belgique

\section{LAURENT DEUTSCH}

Unité européenne d'Eurydice, Bruxelles, Belgique 